\title{
Immunomodulatory effects of bexarotene on tumor-associated macrophages in patients with mycosis fungoides.
}

\section{Introduction and backgrounds}

The immunological background of mycosis fungoides (MF) differs with each tumor stage. As we previously reported, in the early stage, the cancer stroma contains a dense deposition of periostin (POSTN), whereas in the advanced stage, IL-4 expression rather than POSTN deposition is prominent. Since POSTN modulates cell functions including proinflammatory chemokine production, we hypothesized that the immung an inflammatory condition in early stages and a steady state in late stages.

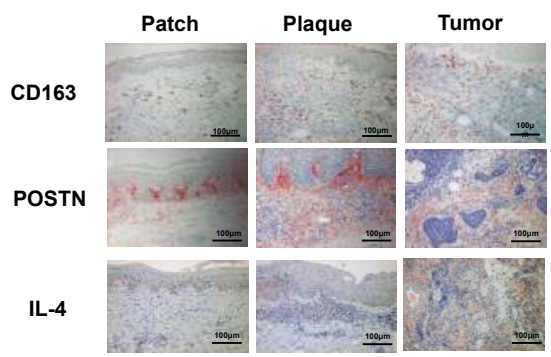

Early stage MF: POSTN>IL-4 Advanced stage MF: POSTN<IL-4

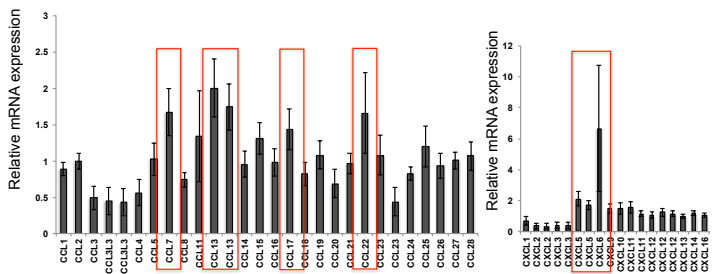

A series of chemokines: CD163+ Macrophages stimulated by POSTN; microarray analysis

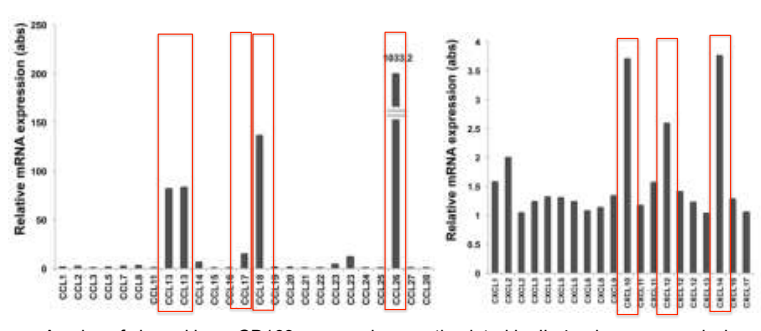

Aseries of chemokines: CD163+ macrophages stimulated by IL-4; microarray analysis

\section{Materials and methods}

Etics

This study was approved by the ethics committee of Tohoku University Graduate School of Medicine, Sendai, Japan (2016-1-650, 2017-1-428, 2017-1-429, ). All patients were given informed consent.

Generation of M2 macrophages from PBMC: for the investigaton of advanced CTCL

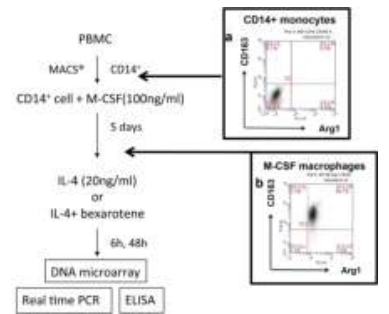

CD14+ cells were isolated from the peripheral blood mononuclear cells (PBMCs) of healthy donors using MACS beads (CD14 microbeads, Miltenyi Biotec, Bergisch Gladbach, Germany) according to the manufacturer's protocol. CD14+ monocytes $\left(2 \times 10^{5} / \mathrm{ml}\right)$ were cultured in complete medium containing $100 \mathrm{ng} / \mathrm{ml}$ of recombinant human M-CSF for 5 days, followed by IL-4 with or without bexarotene.

\section{Results}

Fig. 1: Immunofluorecence staining of advanced CTCL
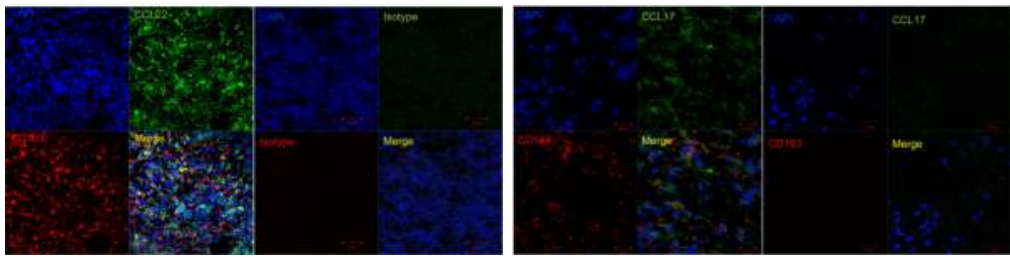

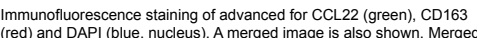
(red) and DAPI (blue, nucleus). A merged image is also shown. Merg
green and red stain yellow. Scale bars, $50 \mathrm{~mm}$. Representative

Immunofluorescence staining of advanced for CCL17 (green), CD163
(red) and DAPI (blue, nucleus). A merged image is also shown. Merged (red) and DAPI (blue, nucleus). A merged image is also shown. Merg
green and red stain yellow. Scale bars, $50 \mathrm{~mm}$. Representative

Fig. 2: Bexarotene reduced the mRNA expression of Th2 chemokines from CD163+ M2 macrophages

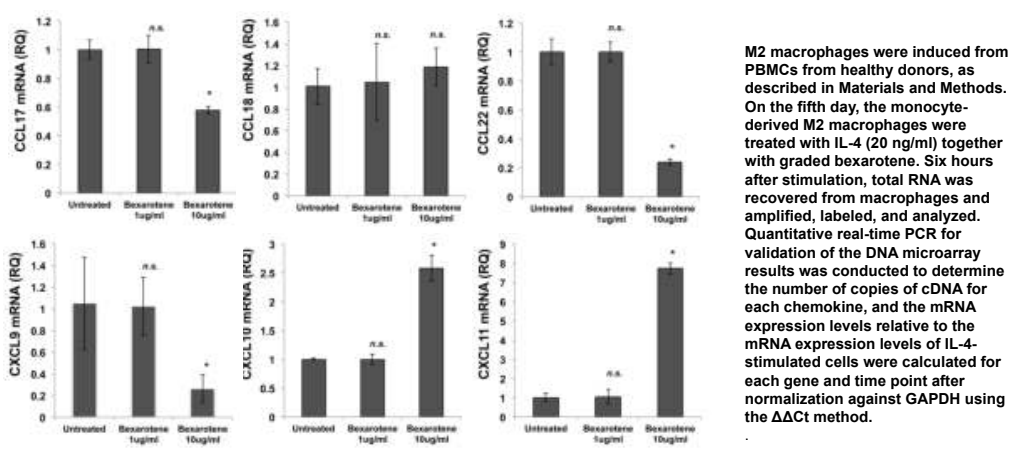

Fig. 3: Bexarotene reduced the production of Th2 chemokines from CD163+ M2 macrophages

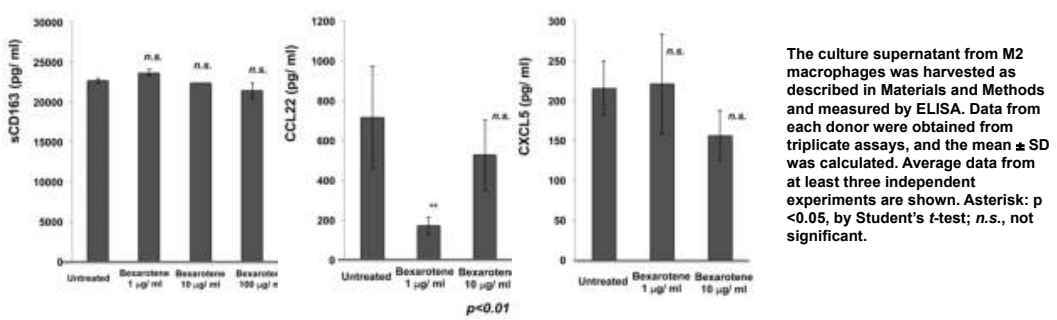

Fig. 4:Serum levels of CCL22 in patients with CTCL: bexarotene reduced serum CCL22 in bexarotene responders
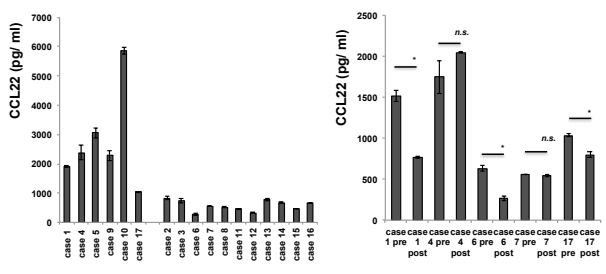

The serum from CTCL patients was donor were obtained from triplicate assays, and the mean \pm SD was calculated. Asterisk: $\mathbf{p}<0.05$, by

\section{SUMMARY}

1. CD163+ TAMs in advanced CTCL expressed CCL22, but merely expressed CCL17.

2. Bexarotene decreased the mRNA expression of Th2 chemokines (CCL17, CCL22) and increased Th1 chemokines (CXCL10, CXCL11) on monocytes-derived CD163+ macrophages in vitro.

Bexarotene did not effect on the activation of $\mathrm{CD} 163+$ macrophages, but decreased the production of CCL22 from CD163+ macrophages in vitro.

4. Serum CCL22 was increased in parallel with the progression of CTCL.

5. Serum levels of CCL22 was decreased in CTCL patients who responded to bexarotene.

References

Furudate $\mathrm{S}$, et al. Tumor-associated $\mathrm{M} 2$ macrophages in mycosis fungoides aquired immunomodulatory function by interferon alpha and interferon gamma. J Dermatol Sci 2016; 83: 182-189.

Furudate $S$, et al. The possible interaction between periostin expressed by cancer stroma and tumor-

associated macrophages in developing mycosis fungoides. Exp Dermatol 2016; 25: 107-112. 